\title{
STABILITY ON COUPLING SIR EPIDEMIC MODEL WITH VACCINATION
}

\author{
HELONG LIU, HOUBAO XU, JINGYUAN YU, AND GUANGTIAN ZHU
}

Received 9 December 2004 and in revised form 21 September 2005

We develop a mathematical model for the disease which can be transmitted via vector and through blood transfusion in host population. The host population is structured by the chronological age. We assume that the instantaneous death and infection rates depend on the age. Applying semigroup theory and so forth, we investigate the existence of equilibria. We also discuss local stability of steady states.

\section{Introduction}

Age-structured epidemic models have been investigated by many authors $[5,6,9,11,15]$. We may find that the epidemic models that most authors discussed mainly include SIS, SIR, and SEIR, that is, the total population of a country or a district was divided into two, three, or four compartments containing susceptibles, exposed, infective, and removed individuals. They have got the threshold conditions for the disease to become endemic, and described the stability of steady-state solutions. However, they did not consider the effects of vector in disease transmission. In fact, many diseases such as malaria, Chagas disease, dengue fever, are transmitted via vector. Therefore, it is necessary and also it is of practical significance to consider the dynamics that includes host population and vector population. So far some authors have studied the mathematical models [2, 3, 7]. Feng et al. in [2] assumed that the population dynamics of malaria and the population genetics of the sickle-cell genes occur on different time scales, formulated vector-host model for malaria, and used the system of ordinary differential equations to describe the model. Inaba and Sekine [7] discussed a vector-host model for the spread of Chagas disease with infection-age.

In this article, we formulate a vector-host model for the disease which is transmitted via vector and through blood transfusion in host population. In order to reflect the fact that the age structure of population affects the dynamics of disease transmission, the host population is structured by the chronological age, and we assume that the instantaneous death and infection rates depend on the age. We consider only a best-case scenario in which vaccine can give life-long immunity for susceptible host population, and assume that removed host population is not infected again. By using the semigroup theory, 
spectrum theory, and so forth, we first prove that the model (2.6)-(2.8) is a well-posed system of differential equations. Second, we get threshold conditions: if the spectral radius of the $T, r(T)$, is less than 1 , the zero solution is the only nonnegative equilibrium point, which corresponds to the disease-free equilibrium point; if $r(T)>1$, there exists a unique positive steady state solution, which corresponds to the endemic equilibrium point. Finally, under the condition given in (4.17), Assumptions 5.1 and 5.6, we get that if $r(T)<1$, the zero solution is locally asymptotically stable; if $r(T)>1$, the zero solution is unstable, and the positive steady state solution is locally asymptotically stable.

\section{The model}

In this section, the host population is divided into three classes: susceptible, infective and removed. Let $s(a, t), i(a, t)$ and $r(a, t)$ be the age-densities of respectively the susceptible, infective, and removed host population at time $t$. We divide the vector population into two groups: susceptible and infective. The vectors is constant, normalized to one, and $v_{0}(t)$ and $v(t)$ are the fractions of susceptible and infective vectors. Let $N(a)$ be the density with respect to age of the total number of the host population. $N(a)$ satisfies

$$
\begin{aligned}
& N(a)=\mu^{*} N e^{-\int_{0}^{a} \mu(\sigma) d \sigma}, \\
& N(a)=s(a, t)+i(a, t)+r(a, t),
\end{aligned}
$$

where $\mu(a)$ denotes the instantaneous death rate at age $a$ of the host population, the constant $N$ is the total size of the host population, $\mu^{*}$ is the crude death rate of the host population. We assume that $\mu(a)$ is nonnegative, locally integrable on $[0,+\infty)$, and satisfies

$$
\int_{0}^{+\infty} \mu(\sigma) d \sigma=+\infty
$$

The crude death rate of the host population is determined such that

$$
\mu^{*} \int_{0}^{+\infty} f(a) d a=1
$$

where $f(a)=e^{-\int_{0}^{a} \mu(\sigma) d \sigma}$ is the survival function. We have the relation

$$
N(a)=\mu^{*} N f(a) .
$$

Let $\delta_{1}$ be the number of bites per vector per unit time and $c$ be the proportion of infected bites hosts that give rise to infection. Then the force of infection for the host population, denoted by $\lambda(a, t)$, is defined by

$$
\lambda(a, t)=\gamma_{1}(a) \int_{0}^{+\infty} \gamma_{2}(a) i(a, t) d a+\eta v
$$

where $\gamma_{1}(a)$ is age-specific infectiousness, $\gamma_{2}(a)$ is age-specific contagion rate, $\gamma_{1}(a), \gamma_{2}(a)$ $\in C[0, \infty)$, and $\gamma_{1}(a), \gamma_{2}(a) \geq 0$ on $[0, \infty), \eta=\delta_{1} c$. Let $\delta=\delta_{1} \delta_{2}$, where $\delta_{2}$ is the proportion of bites to infected hosts that give rise to infection in vector. Then the number of new 
infection of vectors per unit time from infected hosts is given by $\delta_{1} \delta_{2}\left(\int_{0}^{\infty} i(a, t) d a / N\right) v_{0}=$ $\delta\left(\int_{0}^{\infty} i(a, t) d a / N\right) v_{0}$. We let constant $\bar{\mu}$ be the per capita death rate of vectors, $\alpha^{-1}$ denote the average infectious period in the host population, and $p$ be the vaccination rate. Moreover we assume that the death rate of the host population is not affected by the presence of the disease.

With these assumptions, we obtain the following system of equations which describe the dynamics of the vector-host model:

$$
\begin{aligned}
\frac{\partial s(a, t)}{\partial t}+\frac{\partial s(a, t)}{\partial a} & =-(\mu(a)+\lambda(a, t)+\eta v+p) s(a, t) \\
\frac{\partial i(a, t)}{\partial t}+\frac{\partial i(a, t)}{\partial a} & =(\lambda(a, t)+\eta v) s(a, t)-(\mu(a)+\alpha) i(a, t), \\
\frac{\partial r(a, t)}{\partial t}+\frac{\partial r(a, t)}{\partial a} & =p s(a, t)-\mu(a) r(a, t)+\alpha i(a, t), \\
\frac{d v_{0}(t)}{d t} & =\bar{\mu}-\delta \frac{\int_{0}^{+\infty} i(a, t) d a}{N} v_{0}-\bar{\mu} v_{0}, \\
\frac{d v(t)}{d t} & =\delta \frac{\int_{0}^{+\infty} i(a, t) d a}{N} v_{0}-\bar{\mu} v
\end{aligned}
$$

with boundary and initial conditions:

$$
\begin{array}{lll}
s(0, t)=\mu^{*} N, & i(0, t)=0, \quad r(0, t)=0, \\
s(a, 0)=s_{0}(a), \quad i(a, 0)=i_{0}(a), & r(a, 0)=r_{0}(a), \quad v_{0}(0)=v_{0}, \quad v(0)=v_{1},
\end{array}
$$

where

$$
\begin{gathered}
s_{0}(a) \geq 0, \quad i_{0}(a) \geq 0, \quad r_{0}(a) \geq 0, \quad v_{0} \geq 0, \quad v_{1} \geq 0, \\
s_{0}(a)+i_{0}(a)+r_{0}(a)=N(a), \\
v_{0}+v_{1}=1, \\
\lambda(a, t)=\gamma_{1}(a) \int_{0}^{+\infty} \gamma_{2}(b) i(b, t) d b .
\end{gathered}
$$

\section{Existence and uniqueness of solution}

From (2.9), we obtain that $r(a, t), v(t)$ in the system (2.6) can be eliminated. Let $\bar{s}(a, t)=$ $s(a, t)-\mu^{*} N f(a)$, from (2.6)-(2.8) we get the following system:

$$
\begin{aligned}
\frac{\partial \bar{s}(a, t)}{\partial t}+\frac{\partial \bar{s}(a, t)}{\partial a} & =-(\lambda(a, t)+\eta v+p+\mu(a))\left(\mu^{*} N f(a)+\bar{s}(a, t)\right)+\mu^{*} \mu(a) N f(a), \\
\frac{\partial i(a, t)}{\partial t}+\frac{\partial i(a, t)}{\partial a} & =(\lambda(a, t)+\eta v)\left(\mu^{*} N f(a)+\bar{s}(a, t)\right)-(\alpha+\mu(a)) i(a, t), \\
\frac{d v(t)}{d t} & =\frac{\delta \int_{0}^{+\infty} i(a, t) d a}{N}(1-v)-\bar{\mu} v,
\end{aligned}
$$


304 Stability on coupling SIR epidemic model

with boundary and initial conditions:

$$
\begin{gathered}
\bar{s}(0, t)=0, \quad i(0, t)=0, \\
\bar{s}(a, 0)=\bar{s}_{0}(a), \quad i(a, 0)=i_{0}(a), \quad v(0)=v_{1},
\end{gathered}
$$

where $\lambda(a, t)=\gamma_{1}(a) \int_{0}^{+\infty} \gamma_{2}(a) i(a, t) d a$.

We consider the initial-boundary value problem of the system (3.1)-(3.3) as an abstract Cauchy problem:

$$
\begin{aligned}
u^{\prime}(t) & =A u+F(u), \\
u(0) & =\left(\bar{s}_{0}(a), i_{0}(a), v_{1}\right)^{T},
\end{aligned}
$$

where

$$
\begin{gathered}
u=(\bar{s}(a, t), i(a, t), v(t))^{T} \in X, \\
X=L^{1}[0,+\infty) \times L^{1}[0,+\infty) \times R,
\end{gathered}
$$

endowed with the norm

$$
\begin{gathered}
\|x\|=\left\|x_{1}\right\|+\left\|x_{2}\right\|+\left|x_{3}\right|, \quad\left\|x_{i}\right\|=\int_{0}^{+\infty}\left|x_{i}(a)\right| d a, \quad(i=1,2), x=\left(x_{1}, x_{2}, x_{3}\right)^{T} \in X \\
A: D(A) \longrightarrow X \\
A x=\left(\begin{array}{c}
-\left(\frac{d}{d a}+p+\mu(a)\right) x_{1} \\
-\left(\frac{d}{d a}+\alpha+\mu(a)\right) x_{2} \\
-\bar{\mu} x_{3}
\end{array}\right),
\end{gathered}
$$

with domain

$$
D(A)=\left\{\left(x_{1}, x_{2}, x_{3}\right)^{T}, x_{1}, x_{2} \in W_{1}^{1}[0,+\infty), x_{3} \in R,\left(x_{1}(0), x_{2}(0)\right)=(0,0)\right\} .
$$

Suppose $\gamma_{1}(a), \gamma_{2}(a), \mu(a) \in L^{\infty}[0,+\infty)$, we define

$$
F(x)=\left(\begin{array}{c}
F: X \rightarrow X \\
-\left(P\left(x_{2}\right)+\eta x_{3}+p\right) \mu^{*} N f(a)-\left(P\left(x_{2}\right)+\eta x_{3}\right) x_{1} \\
\left(P\left(x_{2}\right)+\eta x_{3}\right)\left(\mu^{*} N f(a)+x_{1}\right) \\
\frac{\delta}{N} H\left(x_{2}\right)\left(1-x_{3}\right)
\end{array}\right),
$$

where $P, H$ is a bounded linear operator on $L^{1}[0,+\infty)$ :

$$
\begin{gathered}
P(\varphi)=\gamma_{1}(a) \int_{0}^{+\infty} \gamma_{2}(b) \varphi(b) d b, \varphi(a) \in L^{1}[0,+\infty), \\
H(\varphi)=\int_{0}^{+\infty} \varphi(a) d a, \varphi(a) \in L^{1}[0,+\infty) .
\end{gathered}
$$


We easily obtain that the operator $A$ is the infinitesimal generator of $C_{0}$-semigroup $T(t), t \geq 0$, and $F$ is continuously Frechet differentiable on $X$. Then for each $u_{0} \in D(A)$, there exists a maximal interval of existence $[0, m)$, and a unique continuous differential solution $t \rightarrow u\left(t, u_{0}\right)$ (see [16]), which satisfies (3.4), where either $m=+\infty$ or $m<+\infty$ and $\lim _{t \rightarrow m}\left\|u\left(t, u_{0}\right)\right\|=+\infty$.

Since $s(a, t)=\mu^{*} N f(a)+\bar{s}(a, t)$, we obtain that the solution $\left(s(a, t), i(a, t), r(a, t), v_{0}(t)\right.$, $v(t))^{T}, t \in[0, m)$ is continuously differentiable and satisfies the system (2.6)-(2.8), where either $m=+\infty$ or $m<\infty$ and $\lim _{t \rightarrow m}\left(\|s(a, t)\|+\|i(a, t)\|+\|r(a, t)\|+\left|v_{0}(t)\right|+|v(t)|\right)=$ $+\infty$. From $\|N(a)\|=\|N(a, t)\|=N$ and $\bar{V}(t)=v_{0}(t)+v(t)=1$, we easily obtain $m=$ $+\infty$.

Thus we have the following result.

Theorem 3.1. The initial-boundary value problem (2.6)-(2.8) has a unique nonnegative classical solution on $X$ with respect to $\left(s_{0}(a), i_{0}(a), r_{0}(a), v_{0}, v_{1}\right)^{T} \in D(A)$.

\section{Existence of steady states}

Let $X^{*}=\left(s^{*}(a), i^{*}(a), r^{*}(a), v_{0}^{*}, v^{*}\right)^{T}$ be the steady state solution of the system (2.6)(2.8). We can obtain:

$$
\begin{aligned}
s^{*}(a) & =\mu^{*} N e^{-\int_{0}^{a}\left(\eta v^{*}+P\left(i^{*}\right)+p+\mu(\tau)\right) d \tau}, \\
i^{*}(a) & =\mu^{*} N e^{-\int_{0}^{a}(\alpha+\mu(s)) d s} \int_{0}^{a}\left(P\left(i^{*}\right)+\eta v^{*}\right) e^{\int_{0}^{\tau}\left(\alpha-\eta v^{*}-P\left(i^{*}\right)-p\right) d s} d \tau, \\
v^{*} & =\frac{\delta H\left(i^{*}\right)}{\delta H\left(i^{*}\right)+N \bar{\mu}},
\end{aligned}
$$

where $\bar{P}\left(i^{*}\right)=\int_{0}^{\infty} \gamma_{2}(a) i^{*}(a) d a, P\left(i^{*}\right)(a)=\gamma_{1}(a) \bar{P}\left(i^{*}\right)$.

Substituting $i^{*}(a)$ into $\bar{P}\left(i^{*}\right)$ and $v^{*}$, we have

$$
\begin{aligned}
\bar{P}\left(i^{*}\right) & =\mu^{*} N \int_{0}^{+\infty} \gamma_{2}(a) e^{-\int_{0}^{a}(\alpha+\mu(s)) d s} \int_{0}^{a}\left(\gamma_{1}(\tau) \bar{P}\left(i^{*}\right)+\eta v^{*}\right) e^{\int_{0}^{\tau}\left(\alpha-p-\gamma_{1}(s) \bar{P}\left(i^{*}\right)-\eta v^{*}\right) d s} d \tau d a, \\
v^{*} & =\frac{\delta \mu^{*} \int_{0}^{+\infty} e^{-\int_{0}^{a}(\alpha+\mu(s)) d s} \int_{0}^{a}\left(\gamma_{1}(\tau) \bar{P}\left(i^{*}\right)+\eta v^{*}\right) e^{\int_{0}^{\tau}\left(\alpha-p-\gamma_{1}(s) \bar{P}\left(i^{*}\right)-\eta v^{*}\right) d s} d \tau d a}{\delta \mu^{*} \int_{0}^{+\infty} e^{-\int_{0}^{a}(\alpha+\mu(s)) d s \int_{0}^{a}\left(\gamma_{1}(\tau) \bar{P}\left(i^{*}\right)+\eta v^{*}\right) e^{\int_{0}^{\tau}\left(\alpha-p-\gamma_{1}(s) \bar{P}\left(i^{*}\right)-\eta v^{*}\right) d s} d \tau d a+\bar{\mu}}} .
\end{aligned}
$$

Let

$$
\begin{gathered}
x=\bar{P}\left(i^{*}\right), \quad y=v^{*} \\
\phi_{1}(\tau)=e^{(\alpha-p) \tau} \int_{\tau}^{+\infty} \gamma_{2}(a) e^{-\int_{0}^{a}(\mu(s)+\alpha) d s} d a \\
\phi_{2}(\tau)=e^{(\alpha-p) \tau} \int_{\tau}^{+\infty} e^{-\int_{0}^{a}(\mu(s)+\alpha) d s} d a .
\end{gathered}
$$


The above representations can be written into

$$
\begin{aligned}
& x=\mu^{*} N \int_{0}^{+\infty}\left(x \gamma_{1}(\tau)+\eta y\right) e^{-\int_{0}^{\tau}\left(x \gamma_{1}(s)+\eta y\right) d s} \phi_{1}(\tau) d \tau \\
& y=\frac{\delta \mu^{*} \int_{0}^{+\infty}\left(x \gamma_{1}(\tau)+\eta y\right) e^{-\int_{0}^{\tau}\left(x \gamma_{1}(s)+\eta y\right) d s} \phi_{2}(\tau) d \tau}{\delta \mu^{*} \int_{0}^{+\infty}\left(x \gamma_{1}(\tau)+\eta y\right) e^{-\int_{0}^{\tau}\left(x \gamma_{1}(s)+\eta y\right) d s} \phi_{2}(\tau) d \tau+\bar{\mu}} .
\end{aligned}
$$

It is clear that one solution of $(4.4)$ is $(x, y)=(0,0)$, which corresponds to the equilibrium point with no disease. In order to get a nontrivial solution of $(4.4)$, we define $F(x, y)$ in $R^{2}$ with the positive cone $R_{+}^{2}$ :

$$
\begin{aligned}
& F(x, y)=\left(\begin{array}{l}
F_{1}(x, y) \\
F_{2}(x, y)
\end{array}\right), \quad(x, y)^{T} \in R_{+}^{2}, \\
& F_{1}(x, y)=\mu^{*} N \int_{0}^{+\infty}\left(x \gamma_{1}(\tau)+\eta y\right) e^{-\int_{0}^{\tau}\left(x \gamma_{1}(s)+\eta y\right) d s} \phi_{1}(\tau) d \tau, \quad(x, y)^{T} \in R_{+}^{2}, \\
& F_{2}(x, y)=\frac{\delta \mu^{*} \int_{0}^{+\infty}\left(x \gamma_{1}(\tau)+\eta y\right) e^{-\int_{0}^{\tau}\left(x \gamma_{1}(s)+\eta y\right) d s} \phi_{2}(\tau) d \tau}{\delta \mu^{*} \int_{0}^{+\infty}\left(x \gamma_{1}(\tau)+\eta y\right) e^{-\int_{0}^{\tau}\left(x \gamma_{1}(s)+\eta y\right) d s} \phi_{2}(\tau) d \tau+\bar{\mu}}, \quad(x, y)^{T} \in R_{+}^{2} .
\end{aligned}
$$

Since the range of $F(x, y)$ is included in $R_{+}^{2}$, and the solutions of (4.4) correspond to fixed points of $F(x, y)$, we can get that the operator $F(x, y)$ has a positive linear majorant $T(x, y)$ defined by:

$$
\begin{aligned}
& T(x, y)=\left(\begin{array}{l}
T_{1}(x, y) \\
T_{2}(x, y)
\end{array}\right), \quad(x, y)^{T} \in R_{+}^{2}, \\
& T_{1}(x, y)=\mu^{*} N \int_{0}^{+\infty}\left(x \gamma_{1}(\tau)+\eta y\right) \phi_{1}(\tau) d \tau, \quad(x, y)^{T} \in R_{+}^{2} \\
& T_{2}(x, y)=\frac{\delta \mu^{*}}{\bar{\mu}} \int_{0}^{+\infty}\left(x \gamma_{1}(\tau)+\eta y\right) \phi_{2}(\tau) d \tau, \quad(x, y)^{T} \in R_{+}^{2} .
\end{aligned}
$$

Let $X^{*}$ be the dual space of $X$. The dual cone $X_{+}^{*}$ is the subset of $X^{*}$ consisting of all positive linear functions on $X$. Let $B(X)$ be the set of bounded linear operators of $X$ into $X . T \in B(X)$ is called positive with respect to the cone $X_{+}$if $T\left(X_{+}\right) \subset X_{+} . S, T \in B(X)$, we say $T \geq S$ if $(T-S) X_{+} \subset X_{+}$. We denote the spectral radius of $T \in B(X)$ by $r(T)$.

Next we introduce the following definition.

Definition 4.1. A positive operator $T \in B(X)$ is called semi-nonsupporting if and only if for every pair $\varphi \in X_{+}-\{0\}, F \in X_{+}^{*}-\{0\}$, there exists a positive integer $p=p(\varphi, F)$ such that $\left\langle F, T^{p} \varphi\right\rangle>0$. A positive operator $T$ is called nonsupporting if and only if for every pair $\varphi \in X_{+}-\{0\}, F \in X_{+}^{*}-\{0\}$, there exists an integer $p=p(\varphi, F)$ such that $\left.\left\langle F, T^{n} \varphi\right\rangle\right\rangle$ 0 holds for all $n \geq p$. 
We need the following lemma (see [13]).

Lemma 4.2. If the cone $X_{+}$is total, $T \in B(X)$ is semi-nonsupporting with respect to $X_{+}$and $r(T)$ is a pole of the resolvent $R(\lambda, T)$. Then the following hold:

(1) $r(T) \in P_{\sigma}(T)-\{0\}, r(T)$ is a simple pole of the resolvent.

(2) The eigenspace corresponding to $r(T)$ is one-dimensional and the corresponding eigenvector $\varphi \in X_{+}$is a nonsupporting point. The relation $T \phi=\lambda \phi$ with $\phi \in X_{+}$implies $\phi=c \varphi$ for some constant $c$.

(3) The eigenspace of $T^{*}$ corresponding to $r(T)$ is also a one-dimensional subspace of $X^{*}$ spanned by a strictly positive functional $F \in X^{*}$.

(4) Assume that $X$ is a Banach lattice. If $T \in B(X)$ is nonsupporting, then the peripheral spectrum of $T$ consists only of $r(T)$, that is, $|\lambda|<r(T)$ for $\lambda \in \sigma(T)-\{r(T)\}$.

Lemma 4.3. The operator $T: X \rightarrow X$ is nonsupporting and compact.

Proof. Let

$$
\begin{array}{cc}
a=\mu^{*} N \int_{0}^{+\infty} \gamma_{1}(\tau) \phi_{1}(\tau) d \tau, & b=\mu^{*} N \eta \int_{0}^{+\infty} \phi_{1}(\tau) d \tau, \\
c=\frac{\delta \mu^{*}}{\bar{\mu}} \int_{0}^{+\infty} \gamma_{1}(\tau) \phi_{2}(\tau) d \tau, \quad d=\frac{\delta \mu^{*} \eta}{\bar{\mu}} \int_{0}^{+\infty} \phi_{2}(\tau) d \tau, \\
m=\min \{a, b, c, d\} .
\end{array}
$$

It is clear that $m>0$ holds. Let

$$
\Psi(x, y)=m\left(\begin{array}{l}
x+y \\
x+y
\end{array}\right), \quad(x, y)^{T} \in R_{+}^{2} .
$$

We can get

$$
T^{n}(x, y) \geq m^{n-1} \Psi(x, y) .
$$

Taking any pair $(x, y)^{T} \in R_{+}^{2}-\{0\}, F \in R_{+}^{2 *}-\{0\}$, we obtain $\left\langle F, T^{n}(x, y)\right\rangle>0$, that is, $T$ is nonsupporting. Since $x, y \in R$, it is clear that $T(x, y)$ is compact.

From Lemma 4.2 we obtain that the spectral radius $r(T)$ of operator $T$ is the only positive eigenvalue with a positive eigenvector and also an eigenvalue of the dual operator $T^{*}$ with a strictly positive eigenfunctional.

TheOREM 4.4. Let $r(T)$ be the spectral radius of the operator $T$. Then the following results hold:

(1) If $r(T) \leq 1$, then $(0,0)^{T}$ is unique nonnegative solution of $(x, y)^{T}=F(x, y)$.

(2) If $r(T)>1$, then $(x, y)^{T}=F(x, y)$ exists at least one non-zero positive solution.

Proof. Suppose $r(T) \leq 1$, we have $T(x, y)-F(x, y) \in R_{+}^{2}-\{0\}$ for $(x, y)^{T} \in R_{+}^{2}-\{0\}$. If there exists a $\left(x_{0}, y_{0}\right)^{T} \in R_{+}^{2}-\{0\}$ which satisfies $\left(x_{0}, y_{0}\right)^{T}=F\left(x_{0}, y_{0}\right)$, then $F\left(x_{0}, y_{0}\right) \leq$ $T\left(x_{0}, y_{0}\right)$. Let $F^{*} \in R_{+}^{2 *}-\{0\}$ be the adjoint eigenvector of $T$ with respect to $r(T)$. Taking 
duality pairing, we get

$$
\left\langle F^{*}, T\left(x_{0}, y_{0}\right)-\left(x_{0}, y_{0}\right)^{T}\right\rangle=\left\langle\left(T^{*}-I^{*}\right) F^{*},\left(x_{0}, y_{0}\right)^{T}\right\rangle=(r(T)-1)\left\langle F^{*},\left(x_{0}, y_{0}\right)^{T}\right\rangle>0 .
$$

Since $T\left(x_{0}, y_{0}\right)-\left(x_{0}, y_{0}\right)=T\left(x_{0}, y_{0}\right)-F\left(x_{0}, y_{0}\right) \in R_{+}^{2}-\{0\}$ and $F^{*}$ is strictly positive, thus we have $r(T)>1$. That is a contradiction. This shows that (1) holds.

It is easily to see $F(x, y)$ is a compact (completely continuous) operator in $R_{+}^{2}$. Moreover, if we define the number $M$ by

$$
M=\max \left\{\mu^{*} N M_{1}, \frac{\delta^{*} \mu^{*}}{\bar{\mu}} M_{2}\right\},
$$

where $M_{1}=\sup _{0 \leq a<\infty} \phi_{1}(a), M_{2}=\sup _{0 \leq a<\infty} \phi_{2}(a)$. Then the set $\Omega=\left\{(x, y)^{T} \in R_{+}^{2}\right.$, $\left.\sqrt{x^{2}+y^{2}} \leq \sqrt{2} M\right\}$ is invariant for $F(x, y)$. In fact, for $(x, y)^{T} \in \Omega$, we have

$$
\begin{gathered}
0 \leq F_{1}(x, y) \leq \mu^{*} N \sup _{0 \leq a<\infty} \phi_{1}(a) \int_{0}^{+\infty}\left(x \gamma_{1}(\tau)+\eta y\right) e^{-\int_{0}^{\tau}\left(x \gamma_{1}(s)+\eta y\right) d s} d \tau=M, \\
0 \leq F_{2}(x, y) \leq \frac{\delta^{*} \mu^{*}}{\bar{\mu}} \sup _{0 \leq a<\infty} \phi_{2}(a) \int_{0}^{+\infty}\left(x \gamma_{1}(\tau)+\eta y\right) e^{-\int_{0}^{\tau}\left(x \gamma_{1}(s)+\eta y\right) d s} d \tau=M .
\end{gathered}
$$

We define $F_{r}$ by

$$
F_{r}(x, y)=\left\{\begin{array}{l}
F(x, y), \quad \sqrt{x^{2}+y^{2}} \geq r,(x, y)^{T} \in R_{+}^{2}, \\
F(x, y)+\left(r-\sqrt{x^{2}+y^{2}}\right)\left(x_{0}, y_{0}\right)^{T}, \quad \sqrt{x^{2}+y^{2}} \leq r,(x, y)^{T} \in R_{+}^{2},
\end{array}\right.
$$

where $\left(x_{0}, y_{0}\right)^{T}$ is the positive eigenvector of $T$ corresponding to $r(T)>1$. It is easy to get that $F_{r}(x, y)$ is also compact (completely continuous) and transforms the set $\Omega_{r}=$ $\left\{(x, y)^{T} \in R_{+}^{2}, \sqrt{x^{2}+y^{2}} \leq M+r \sqrt{x_{0}^{2}+y_{0}^{2}}\right\}$ into itself. Because $\Omega_{r}$ is bounded, convex and closed in $R^{2}$, from Schauder's fixed point theorem we have that $F_{r}(x, y)$ has a fixed point $\left(x_{1}, y_{1}\right)^{T} \in \Omega_{r}$. Note that the Frechet derivation of $F(x, y)$ at $(0,0)^{T}$ is $T$ and $T$ does not have in $R_{+}^{2}$ eigenvector corresponding to eigenvalue one. We use the method of Theorem 4.11 (see [8]) and it can be shown that the norms of these fixed points are greater than $r$ if $r$ is sufficiently small. That is, $F(x, y)$ has a positive fixed point.

Now we introduce conception of concave operator.

Definition 4.5. $X_{+}$is a positive cone in a real Banach space $X$ and $\leq$ is the partial ordering defined by $X_{+}$. A positive operator $A: X_{+} \rightarrow X_{+}$is called a concave operator if there exists a $x_{0} \in X_{+}-\{0\}$ which satisfies the following conditions:

(1) For any $x \in X_{+}-\{0\}$ there exists $\alpha=\alpha(x)>0$ and $\beta=\beta(x)>0$ such that $\alpha x_{0} \leq$ $A x \leq \beta x_{0}$, that is, $A x$ is comparable with $x_{0}$.

(2) $A(t x) \geq t A x$ for $0 \leq t \leq 1$ and for every $x \in X_{+}$such that

$$
\alpha x_{0} \leq x \leq \beta x_{0}
$$


Lemma 4.6 [8]. Suppose that the operator $A: X_{+} \rightarrow X_{+}$is monotone and concave. If for any $x \in X_{+}$satisfying $\alpha x_{0} \leq x \leq \beta x_{0}(\alpha=\alpha(x)>0, \beta=\beta(x)>0)$ and $0<t<1$, there exists $\eta=\eta(x, t)$ such that

$$
A(t x) \geq t A x+\eta x_{0}
$$

then A exists at most a positive fixed point.

THEOREM 4.7. If the following condition is satisfied

$$
(p-\alpha) \int_{\tau}^{\infty} \gamma_{2}(a) e^{-\int_{0}^{a}(\mu(s)+\alpha) d s} d a+\gamma_{2}(\tau) e^{-\int_{0}^{\tau}(\mu(s)+\alpha) d s} \geq 0, \quad \tau \geq 0
$$

and $r(T)>1$, then $F(x, y)$ has one unique positive fixed point.

Proof. We first prove that $F(x, y)$ is a monotone operator in $R_{+}^{2}$. From (4.5), we have

$$
\begin{aligned}
& F_{1}(x, y)=-\mu^{*} N \int_{0}^{+\infty}\left[-\left(x \gamma_{1}(\tau)+\eta y\right) e^{-\int_{0}^{\tau}\left(x \gamma_{1}(s)+\eta y\right) d s}\right] e^{(\alpha-p) \tau} \\
& \cdot \int_{\tau}^{+\infty} \gamma_{2}(a) e^{-\int_{0}^{a}(\mu(s)+\alpha) d s} d a d \tau \\
& =\mu^{*} N \int_{0}^{+\infty} \gamma_{2}(a) e^{-\int_{0}^{a}(\mu(s)+\alpha) d s} d a \\
& -(p-\alpha) \mu^{*} N \int_{0}^{+\infty} e^{-\int_{0}^{\tau}\left(x \gamma_{1}(s)+\eta y\right) d s} e^{(\alpha-p) \tau} \int_{\tau}^{+\infty} \gamma_{2}(a) e^{-\int_{0}^{a}(\mu(s)+\alpha) d s} d a d \tau \\
& -\mu^{*} N \int_{0}^{+\infty} \gamma_{2}(\tau) e^{-\int_{0}^{\tau}\left(x \gamma_{1}(s)+\eta y\right) d s} e^{-\int_{0}^{\tau}(\mu(s)+p) d s} d \tau, \\
& F_{2}(x, y)=\frac{\delta \mu^{*} \int_{0}^{+\infty}\left[-\left(x \gamma_{1}(\tau)+\eta y\right) e^{-\int_{0}^{\tau}\left(x \gamma_{1}(s)+\eta y\right) d s}\right] \phi_{2}(\tau) d \tau}{\delta \mu^{*} \int_{0}^{+\infty}\left[-\left(x \gamma_{1}(\tau)+\eta y\right) e^{-\int_{0}^{\tau}\left(x \gamma_{1}(s)+\eta y\right) d s}\right] \phi_{2}(\tau) d \tau+\bar{\mu}} \\
& =1-\frac{1}{\delta \mu^{*} \int_{0}^{+\infty}\left[-\left(x \gamma_{1}(\tau)+\eta y\right) e^{-\int_{0}^{\tau}\left(x \gamma_{1}(s)+\eta y\right) d s}\right] \phi_{2}(\tau) d \tau+\bar{\mu}} \\
& =1-\frac{1}{\bar{\mu}+\Pi_{1}-\Pi_{2}(x, y)-\Pi_{3}(x, y)}, \\
& \Pi_{1}=\mu^{*} \int_{0}^{+\infty} e^{-\int_{0}^{a}(\mu(s)+\alpha) d s} d a \\
& \Pi_{2}(x, y)=(p-\alpha) \mu^{*} \int_{0}^{+\infty} e^{-\int_{0}^{\tau}\left(x \gamma_{1}(s)+\eta y\right) d s} \phi_{2}(\tau) d \tau, \\
& \Pi_{3}(x, y)=\delta \mu^{*} \int_{0}^{+\infty} e^{-\int_{0}^{\tau}\left(x \gamma_{1}(s)+\eta y\right) d s} e^{-\int_{0}^{\tau}(p+\mu(s)) d s} d \tau .
\end{aligned}
$$

If the inequality (4.17) holds, it is clear that $F_{1}(x, y), F_{2}(x, y)$ are increasing for $(x, y)^{T} \in$ $R_{+}^{2}$. We conclude that $F(x, y)$ is increasing for $(x, y)^{T} \in R_{+}^{2}$. 
310 Stability on coupling SIR epidemic model

Let

$$
\begin{aligned}
u_{0} & =(1,1)^{T}, \\
\alpha(x, y) & =\min \left\{\alpha_{1}(x, y), \alpha_{2}(x, y), \beta_{1}(x, y), \beta_{2}(x, y)\right\}, \\
\beta(x, y) & =\max \left\{\alpha_{1}(x, y), \alpha_{2}(x, y), \beta_{1}(x, y), \beta_{2}(x, y)\right\}, \\
\alpha_{1}(x, y) & =\mu^{*} N \int_{0}^{+\infty} x \gamma_{1}(\tau) e^{-\int_{0}^{\tau}\left(x \gamma_{1}(s)+\eta y\right) d s} \phi_{1}(\tau) d \tau, \\
\alpha_{2}(x, y) & =\mu^{*} N \int_{0}^{+\infty} \eta y e^{-\int_{0}^{\tau}\left(x \gamma_{1}(s)+\eta y\right) d s} \phi_{1}(\tau) d \tau, \\
\beta_{1}(x, y) & =\frac{\delta \mu^{*} \int_{0}^{+\infty} x \gamma_{1}(\tau) e^{-\int_{0}^{\tau}\left(x \gamma_{1}(s)+\eta y\right) d s} \phi_{2}(\tau) d \tau}{\delta \mu^{*} \int_{0}^{+\infty}\left[-\left(x \gamma_{1}(\tau)+\eta y\right) e^{\left.-\int_{0}^{\tau}\left(x \gamma_{1}(s)+\eta y\right) d s\right] \phi_{2}(\tau) d \tau+\bar{\mu}},\right.} \\
\beta_{2}(x, y) & =\frac{\delta \mu^{*} \int_{0}^{+\infty} \eta y e^{-\int_{0}^{\tau}\left(x \gamma_{1}(s)+\eta y\right) d s} \phi_{2}(\tau) d \tau}{\delta \mu^{*} \int_{0}^{+\infty}\left[-\left(x \gamma_{1}(\tau)+\eta y\right) e^{\left.-\int_{0}^{\tau}\left(x \gamma_{1}(s)+\eta y\right) d s\right] \phi_{2}(\tau) d \tau+\bar{\mu}} .\right.}
\end{aligned}
$$

We have $\alpha(x, y) u_{0} \leq F(x, y) \leq \beta(x, y) u_{0}$, and easily get $F(t x, t y) \geq t F(x, y), 0<t<1$. From Definition 4.5 , we obtain that $F(x, y)$ is a concave operator.

Next we prove that $F(x, y)$ satisfies the condition (4.16).

From (4.18), we get

$$
\begin{aligned}
F_{1}(t x, t y)= & \mu^{*} N t \int_{0}^{+\infty}\left(x \gamma_{1}(\tau)+\eta y\right) e^{-t \int_{0}^{\tau}\left(x \gamma_{1}(s)+\eta y\right) d s} \phi_{1}(\tau) d \tau \\
& -\mu^{*} N \int_{0}^{+\infty}\left(x \gamma_{1}(\tau)+\eta y\right) e^{-\int_{0}^{\tau}\left(x \gamma_{1}(s)+\eta y\right) d s} \phi_{1}(\tau) d \tau \\
= & \eta_{1}(x, y, t)>0 .
\end{aligned}
$$

Let

$$
j(t)=\frac{\delta \mu^{*} \int_{0}^{+\infty}\left(x \gamma_{1}(\tau)+\eta y\right) e^{-t \int_{0}^{\tau}\left(x \gamma_{1}(s)+\eta y\right) d s} \phi_{2}(\tau) d \tau}{t \delta \mu^{*} \int_{0}^{+\infty}\left(x \gamma_{1}(\tau)+\eta y\right) e^{-t \int_{0}^{\tau}\left(x \gamma_{1}(s)+\eta y\right) d s} \phi_{2}(\tau) d \tau+\bar{\mu}} .
$$

It is easy to prove that $j(t)$ is decreasing in $0<t<1,(x, y)^{T} \in R_{+}^{2}$. So, we get

$$
F_{2}(t x, t y)-t F_{2}(x, y)=t(j(t)-j(1))=\eta_{2}(x, y, t)>0 .
$$

Let

$$
\eta(x, y, t)=\left(\begin{array}{l}
\eta_{1}(x, y, t) \\
\eta_{2}(x, y, t)
\end{array}\right)
$$

We obtain that $F(x, y)$ satisfies the condition (4.16). From Lemma 4.6, we get $F(x, y)$ has only a positive fixed point. This theorem is proved. 
In the following, we will show that there exists $p>0$ such that the inequality (4.17) and $r(T)>1$ hold. In the case $\gamma_{1}(a) \equiv \gamma_{1}, \gamma_{2}(a) \equiv \gamma_{2}, \mu(a) \equiv \mu$, we have

$$
\begin{aligned}
(p-\alpha) & \int_{\tau}^{\infty} \gamma_{2}(a) e^{-\int_{0}^{a}(\mu(s)+\alpha) d s} d a+\gamma_{2}(\tau) e^{-\int_{0}^{\tau}(\mu(s)+\alpha) d s} \\
& =\left(\frac{p \gamma_{2}}{\mu+\alpha}-\frac{\alpha \gamma_{2}}{\mu+\alpha}+\gamma_{2}\right) e^{-(\mu+\alpha) \tau} \\
& \geq \frac{p \gamma_{2}}{\mu+\alpha} e^{-(\mu+\alpha) \tau} .
\end{aligned}
$$

So, the inequality (4.17) holds for $p \geq 0$. From (4.6), we get

$$
\begin{aligned}
& T_{1}(x, y)=\frac{\mu^{*} N \gamma_{1} \gamma_{2}}{(\mu+\alpha)(\mu+p)} x+\frac{\mu^{*} N \eta \gamma_{2}}{(\mu+\alpha)(\mu+p)} y, \\
& T_{2}(x, y)=\frac{\delta \mu^{*} \gamma_{1}}{\bar{\mu}(\mu+p)} x+\frac{\delta \mu^{*} \eta}{\bar{\mu}(\mu+p)} y, \\
& T(x, y)=\left(\begin{array}{cc}
\frac{\mu^{*} N \gamma_{1} \gamma_{2}}{(\mu+\alpha)(\mu+p)} & \frac{\mu^{*} N \eta \gamma_{2}}{(\mu+\alpha)(\mu+p)} \\
\frac{\delta \mu^{*} \gamma_{1}}{\bar{\mu}(\mu+p)} & \frac{\delta \mu^{*} \eta}{\bar{\mu}(\mu+p)}
\end{array}\right)\left(\begin{array}{l}
x \\
y
\end{array}\right), \quad(x, y)^{T} \in R_{+}^{2} .
\end{aligned}
$$

We get the eigenvalues of $T: \lambda_{1}=0, \lambda_{2}=\mu^{*} \delta \eta / \bar{\mu}(\mu+p)+\mu^{*} N \gamma_{1} \gamma_{2} /(\mu+\alpha)(\mu+p)$. Thus $r(T)=\lambda_{2}$. If $\mu^{*} \delta \eta / \bar{\mu} \mu+\mu^{*} N \gamma_{1} \gamma_{2} /(\mu+\alpha) \mu>1$, then there exists $p>0$ such that $r(T)>1$ holds.

\section{Local stability of equilibria}

Since $r(a, t)=N(a)-s(a, t)-i(a, t), v_{0}(t)=1-v(t)$, it is sufficient to consider the system (2.6)-(2.8) in terms of only $s(a, t), i(a, t), v(t)$. Writing the solution of the system (2.6)(2.8) in the form $s(a, t)=s^{*}(a)+x(a, t), i(a, t)=i^{*}(a)+y(a, t), v=v^{*}+z(t)$, we get a linearized system around the equilibrium $\left(s^{*}(a), i^{*}(a), v^{*}\right)^{T}$ :

$$
\begin{aligned}
\frac{\partial x(a, t)}{\partial t}+\frac{\partial x(a, t)}{\partial a} & =-\left(P\left(i^{*}\right)(a)+\eta v^{*}+p+\mu(a)\right) x-(\lambda(a, t)+\eta z) s^{*}(a), \\
\frac{\partial y(a, t)}{\partial t}+\frac{\partial y(a, t)}{\partial a} & =\left(P\left(i^{*}\right)(a)+\eta v^{*}\right) x-(\alpha+\mu(a)) y+(\lambda(a, t)+\eta z) s^{*}, \\
\frac{d z(t)}{d t} & =-\left(\delta H\left(i^{*}\right)+\bar{\mu}\right) z+\delta H(y)\left(1-v^{*}\right),
\end{aligned}
$$

with boundary and initial conditions:

$$
\begin{gathered}
x(0, t)=0, \quad y(0, t)=0, \\
x(a, 0)=0, \quad y(a, 0)=0, \quad z(0)=0,
\end{gathered}
$$

where

$$
\lambda(a, t)=\gamma_{1}(a) \int_{0}^{+\infty} \gamma_{2}(a) y(a, t) d a .
$$


312 Stability on coupling SIR epidemic model

We rewrite the initial-boundary value problem (5.1) and (5.2) as an abstract Cauchy problem:

$$
\frac{d \Psi(t)}{d t}=B \Psi(t)+C \Psi(t), \quad \Psi(0)=\Psi_{0}
$$

where

$$
\begin{gathered}
\Psi=(x, y, z)^{T} \in X, \quad B: D(B) \longrightarrow X \\
B U=\left(\begin{array}{c}
-\left(\frac{\partial}{\partial a}+P\left(i^{*}\right)(a)+\eta v^{*}+p+\mu(a)\right) u_{1} \\
-\left(\frac{\partial}{\partial a}+\alpha+\mu(a)\right) u_{2}+\left(P\left(i^{*}\right)(a)+\eta v^{*}\right) u_{1} \\
-\left(\delta H\left(i^{*}\right)+\bar{\mu}\right) u_{3}
\end{array}\right),
\end{gathered}
$$

with domain

$$
\begin{gathered}
D(B)=\left\{\left(u_{1}, u_{2}, u_{3}\right)^{T}, u_{1}, u_{2} \in W_{1}^{1}[0,+\infty), u_{3} \in R, u_{1}(0)=0, u_{2}(0)=0\right\}, \\
C: X \longrightarrow X, \quad C=\left(\begin{array}{c}
-\left(\lambda(a, t)+\eta u_{3}\right) s^{*}(a) \\
\left(\lambda(a, t)+\eta u_{3}\right) s^{*}(a) \\
\delta H\left(u_{2}\right)\left(1-v^{*}\right)
\end{array}\right), \quad D(C)=X .
\end{gathered}
$$

In the following, we make an assumption.

Assumption 5.1. (1) $\gamma_{1}(a) \in C[0, \infty)$ is uniformly continuous.

(2) There exists a $M_{3}>0$ such that $\gamma_{1}(a), \gamma_{2}(a)<M_{3}$ holds, for $a \in[0,+\infty)$.

We introduce the following lemma (see [10]).

Lemma 5.2. Suppose that $X$ is a Banach lattice. Let $S, T$ be positive operator in $B(X)$. The following results hold:

(1) If $S \leq T$, then $r(S) \leq r(T)$.

(2) If $S, T$ are semi-nonsupporting operators, then $S \leq T, S \neq T$ implies that $r(S)<$ $r(T)$ holds.

Now let us consider the characteristic equation of $B+C$ :

$$
\lambda U=(B+C) U, \quad U=\left(u_{1}, u_{2}, u_{3}\right) \in D(B), \lambda \in C .
$$

We obtain

$$
\begin{aligned}
\frac{d u_{1}(a)}{d a} & =-\left(\lambda+P\left(i^{*}\right)(a)+\eta v^{*}+p+\mu(a)\right) u_{1}-\left(P\left(u_{2}\right)(a)+\eta u_{3}\right) s^{*}(a), \\
\frac{d u_{2}(a)}{d a} & =\left(P\left(i^{*}\right)(a)+\eta v^{*}\right) u_{1}-(\lambda+\alpha+\mu(a)) u_{2}+\left(P\left(u_{2}\right)(a)+\eta u_{3}\right) s^{*}(a), \\
\lambda u_{3} & =-\left(\delta H\left(i^{*}\right)+\hat{\mu}\right) u_{3}+\left(1-v^{*}\right) \delta H\left(u_{2}\right),
\end{aligned}
$$

with initial conditions

$$
u_{1}(0)=0, \quad u_{2}(0)=0 .
$$


From (5.8) and (5.9), we have

$$
\begin{aligned}
u_{1}(a)= & -\mu^{*} N e^{-\int_{0}^{a}\left(\lambda+P\left(i^{*}\right)(s)+\eta v^{*}+p+\mu(s)\right) d s} \int_{0}^{a}\left(\gamma_{1}(s) \bar{P}\left(u_{2}\right)+\eta u_{3}\right) e^{\lambda s} d s, \\
u_{2}(a)= & e^{-\int_{0}^{a}(\lambda+\alpha+\mu(s)) d s} \int_{0}^{a}\left[\left(\gamma_{1}(s) \bar{P}\left(u_{2}\right)+\eta u_{3}\right) s^{*}(s)+\left(P\left(i^{*}\right)(s)+\eta v^{*}\right) u_{1}(s)\right] \\
& \cdot e^{\int_{0}^{s}\left(\lambda+\alpha+\mu^{*}(\tau)\right) d \tau} d s \\
= & \mu^{*} N e^{-\int_{0}^{a}(\lambda+\alpha+\mu(s)) d s} \int_{0}^{a}\left(\gamma_{1}(s) \bar{P}(s)+\eta u_{3}\right) e^{\int_{0}^{s}\left(\lambda+\alpha-P\left(i^{*}\right)(\tau)-\eta v^{*}-p\right) d \tau} d s \\
& -\mu^{*} N e^{-\int_{0}^{a}(\lambda+\alpha+\mu(s)) d s} \int_{0}^{a}\left(P\left(i^{*}\right)(s)+\eta v^{*}\right) e^{\int_{0}^{s}\left(\alpha-P\left(i^{*}\right)(\tau)-\eta v^{*}-p\right) d \tau} \\
& \cdot \int_{0}^{s}\left(\gamma_{1}(\tau) \bar{P}(\tau)+\eta u_{3}\right) e^{\lambda \tau} d \tau d s, \\
u_{3}= & \frac{\left(1-v^{*}\right) \delta H\left(u_{2}\right)}{\lambda+\delta H(i *)+\bar{\mu}} .
\end{aligned}
$$

Let $Y=\left\{x_{1} \gamma_{1}(a)+\eta x_{2}, x_{1}, x_{2} \in R\right\}$ with norm

$$
\|\bar{x}\|=\max _{a \in[0,+\infty)}|\bar{x}|, \quad \bar{x}=x_{1} \gamma_{1}(a)+\eta x_{2} \in Y .
$$

Then $Y$ is complete Banach space.

Substituting $u_{2}(a)$ into $\bar{P}\left(u_{2}\right), u_{3}$, we get

$$
\begin{aligned}
\theta(a)= & \mu^{*} N \gamma_{1}(a) \int_{0}^{+\infty} \gamma_{2}(a) E(a) \int_{0}^{a} \theta(s) e^{\int_{0}^{s}\left(\lambda+\alpha-P\left(i^{*}\right)(\tau)-\eta v^{*}-p\right) d \tau} d s d a \\
& -\mu^{*} N \gamma_{1}(a) \int_{0}^{+\infty} \gamma_{2}(a) E(a) \int_{0}^{a}\left(P\left(i^{*}\right)(s)+\eta v^{*}\right) e^{\int_{0}^{s}\left(\alpha-P\left(i^{*}\right)(\tau)-\eta v^{*}-p\right) d \tau} \\
& +\int_{0}^{s} \theta(\sigma) e^{\lambda \sigma} d \sigma d s d a \\
& \frac{\left(1-v^{*}\right) \delta \eta \mu^{*} N}{\lambda+\delta H\left(i^{*}\right)+\bar{\mu}}\left[\int_{0}^{+\infty} E(a) \int_{0}^{a} \theta(s) e^{\int_{0}^{s}\left(\lambda+\alpha-P\left(i^{*}\right)(\tau)-\eta v^{*}-p\right) d \tau} d s d a\right. \\
-\int_{0}^{+\infty} E(a) \int_{0}^{a}\left(P\left(i^{*}\right)(s)+\eta v^{*}\right) e^{\int_{0}^{s}\left(\alpha-P\left(i^{*}\right)(\tau)-\eta v^{*}-p\right) d \tau} & \left.\cdot \int_{0}^{s} \theta(\sigma) e^{\lambda \sigma} d \sigma d s d a\right],
\end{aligned}
$$

where $E(a)=e^{-\int_{0}^{a}(\lambda+\alpha+\mu(s)) d s}, \theta(a)=\gamma_{1}(a) \bar{p}\left(u_{2}\right)+\eta u_{3}$.

If $\lambda$ is an eigenvalue of $B+C$, then there exists $\theta \neq 0 \in Y$ such that

$$
\theta=T_{\lambda}(\theta)
$$

where $T_{\lambda}(\theta)$ denote the right-hand side of (5.12). 


\section{Stability on coupling SIR epidemic model}

So we investigate $\lambda$ such that the eigenvalue of $T_{\lambda}$ is 1 . Let $\delta^{*}=\delta H\left(i^{*}\right)+\bar{\mu}$. If $\alpha \geq \delta^{*}$, as $\lambda \in\left(-\delta^{*},+\infty\right)$ we have

$$
\begin{aligned}
T_{\lambda}(\theta)= & (p-\alpha) \mu^{*} N \gamma_{1}(a) \int_{0}^{+\infty} \theta(s) e^{\int_{0}^{s}\left(\alpha-P\left(i^{*}\right)(\tau)-\eta v^{*}-p\right) d \tau} \int_{0}^{s} \theta(\tau) e^{\lambda \tau} d \tau \\
& \cdot \int_{s}^{+\infty} \gamma_{2}(a) E(a) d a d s \\
+ & \gamma_{1}(a) \mu^{*} N \int_{0}^{+\infty} \gamma_{2}(s) E(s) e^{\int_{0}^{s}\left(\alpha-P\left(i^{*}\right)(\tau)-\eta v^{*}-p\right) d \tau} \int_{0}^{s} \theta(\tau) e^{\lambda \tau} d \tau d s \\
+ & \frac{\eta \delta \mu^{*} N\left(1-v^{*}\right)}{\lambda+\delta^{*}}\left[(p-\alpha) \int_{0}^{+\infty} \theta(s) e^{\int_{0}^{s}\left(\alpha-P\left(i^{*}\right)(\tau)-\eta v^{*}-p\right) d \tau} \int_{0}^{s} \theta(\tau) e^{\lambda \tau} d \tau\right. \\
& \cdot \int_{s}^{+\infty} E(a) d a d s \\
& \left.+\int_{0}^{+\infty} E(s) e^{\int_{0}^{s}\left(\alpha-P\left(i^{*}\right)(\tau)-\eta v^{*}-p\right) d \tau} \int_{0}^{s} \theta(\tau) e^{\lambda \tau} d \tau d s\right] .
\end{aligned}
$$

Using (4.17), it is clear to see that $T_{\lambda}(\theta)$ is decreasing as a function of $\lambda \in\left(-\delta^{*},+\infty\right)$, $\theta \in Y_{+}$. From Assumption 5.1, we get that $T_{\lambda}(\theta), \lambda \in\left(-\delta^{*},+\infty\right)$, is compact and nonsupporting.

From (5.14), we have

$$
\begin{aligned}
& T_{\lambda}(\theta) \geq \frac{\eta \delta \mu^{*} N\left(1-v^{*}\right)}{\lambda+\delta^{*}}\left[(p-\alpha) \int_{0}^{+\infty} e^{\int_{0}^{s}\left(\alpha-P\left(i^{*}\right)(\tau)-\eta v^{*}-p\right) d \tau} \int_{0}^{s} \theta(\tau) e^{\lambda \tau} d \tau\right. \\
& \cdot \int_{s}^{+\infty} E(a) d a d s+\int_{0}^{+\infty} E(s) e^{\int_{0}^{s}\left(\alpha-P\left(i^{*}\right)(\tau)-\eta v^{*}-p\right) d \tau} \\
& \left.\cdot \int_{0}^{s} \theta(\tau) e^{\lambda \tau} d \tau d s\right] \\
& =\frac{\eta \delta \mu^{*} N\left(1-v^{*}\right)}{\lambda+\delta^{*}}\left\langle G_{\lambda}, \theta\right\rangle \cdot 1, \quad \lambda \in\left(-\delta^{*},+\infty\right), \\
& \left\langle G_{\lambda}, \theta\right\rangle=(p-\alpha) \int_{0}^{+\infty} e^{\int_{0}^{s}\left(\alpha-P\left(i^{*}\right)(\tau)-\eta v^{*}-p\right) d \tau} \int_{0}^{s} \theta(\tau) e^{\lambda \tau} d \tau \int_{s}^{+\infty} E(a) d a d s \\
& +\int_{0}^{+\infty} E(s) e^{\int_{0}^{s}\left(\alpha-P\left(i^{*}\right)(\tau)-\eta v^{*}-p\right) d \tau} \int_{0}^{s} \theta(\tau) e^{\lambda \tau} d \tau d s, \quad \theta \in Y_{+} .
\end{aligned}
$$

Taking duality pairing with the eigenfunctional $F_{\lambda}$ of $T_{\lambda}$ that corresponds to $r\left(T_{\lambda}\right)$, we obtain

$$
r\left(T_{\lambda}\right)\left\langle F_{\lambda}, \theta\right\rangle \geq \frac{\eta \delta \mu^{*} N\left(1-v^{*}\right)}{\lambda+\delta^{*}}\left\langle G_{\lambda}, \theta\right\rangle\left\langle F_{\lambda}, 1\right\rangle .
$$

If taking $\theta=1$, we have

$$
r\left(T_{\lambda}\right) \geq \frac{\eta \delta \mu^{*} N\left(1-v^{*}\right)}{\lambda+\delta^{*}}\left\langle G_{\lambda}, 1\right\rangle .
$$


If $\lambda \in\left(-\delta^{*},+\infty\right)$, then

$$
0<\left\langle G_{\lambda}, 1\right\rangle<+\infty, \quad \lim _{\lambda \rightarrow-\delta^{*}} r\left(T_{\lambda}\right)=+\infty .
$$

We can also prove that if $\alpha<\delta^{*}$, then there exists $-\infty<\alpha^{*}<0$ such that

$$
\lim _{\lambda \rightarrow \alpha^{*}} r\left(T_{\lambda}\right)=+\infty
$$

From (5.14), we can obtain

$$
\begin{aligned}
T_{\lambda}(\theta) \leq & \gamma_{1}(a) \mu^{*} N \int_{0}^{+\infty} \gamma_{2}(a) e^{-(\lambda+\alpha) a} \int_{0}^{a} \theta(\tau) e^{\int_{0}^{\tau}\left(\lambda+\alpha-P\left(i^{*}\right)(s)-\eta v^{*}-p\right) d s} d \tau d a \\
& +\frac{\eta \delta \mu^{*} N\left(1-v^{*}\right)}{\lambda+\delta^{*}} \int_{0}^{+\infty} e^{-(\lambda+\alpha) a} \int_{0}^{a} \theta(\tau) e^{\int_{0}^{\tau}\left(\lambda+\alpha-P\left(i^{*}\right)(s)-\eta v^{*}-p\right) d s} d \tau d a \\
< & \left(M_{3}^{2}+\frac{\eta \delta\left(1-v^{*}\right)}{\lambda+\delta^{*}}\right) \mu^{*} N \int_{0}^{+\infty} e^{-\lambda a} \int_{0}^{a} \theta(\tau) e^{\lambda \tau} d \tau d a, \quad \theta \in Y_{+} .
\end{aligned}
$$

Let

$$
\left\langle G_{\lambda}^{*}, \theta\right\rangle=\left(M_{3}^{2}+\frac{\eta \delta\left(1-v^{*}\right)}{\lambda+\delta^{*}}\right) \mu^{*} N \int_{0}^{+\infty} e^{-\lambda a} \int_{0}^{a} \theta(\tau) e^{\lambda \tau} d \tau d a .
$$

We have

$$
\begin{gathered}
T_{\lambda}(\theta) \leq\left\langle G_{\lambda}^{*}, \theta\right\rangle \cdot 1, \quad \theta \in Y_{+}, \\
r\left(T_{\lambda}\right) \leq\left\langle G_{\lambda}^{*}, 1\right\rangle, \\
\lim _{\lambda \rightarrow+\infty} r\left(T_{\lambda}\right) \leq \lim _{\lambda \rightarrow+\infty}\left\langle G_{\lambda}^{*}, 1\right\rangle=0 .
\end{gathered}
$$

From (5.14), we get that $T_{\lambda}(\theta)$ is decreasing as a function of $\lambda \in\left(\delta^{*},+\infty\right)$ or $\left(\alpha^{*},+\infty\right)$, $\theta \in Y_{+}$.

Using Lemma 5.2, we know that the function $\lambda \rightarrow r\left(T_{\lambda}\right)$ is strictly decreasing. If there exists $\lambda \in\left(\delta^{*},+\infty\right)$ or $\left(\alpha^{*},+\infty\right)$ such that $r\left(T_{\lambda}\right)=1$, then $\lambda \in P_{\sigma}(B+C)$. So, we get the following lemma.

Lemma 5.3. If the inequality (4.17) holds, under Assumption 5.1, there exists unique $\lambda_{0} \in$ $\left(\delta^{*},+\infty\right) \cap P_{\sigma}(B+C)$ or $\left(\alpha^{*},+\infty\right) \cap P_{\sigma}(B+C)$ such that the following results hold:
(1) If $r\left(T_{0}\right)>1$, then $\lambda_{0}>0$.
(2) If $r\left(T_{0}\right)=1$, then $\lambda_{0}=0$.
(3) If $r\left(T_{0}\right)<1$, then $\lambda_{0}<0$.

Using the similar argument as [4, Theorem 6.13], we can prove that $\lambda_{0}$ is a dominant singular point. We have the following lemma.

LEMmA 5.4. Under the condition of Lemma 5.3, if there exists a $\lambda, r\left(T_{\lambda}\right)=1, \lambda \neq \lambda_{0}$, then $\operatorname{Re} \lambda<\lambda_{0}$.

We define

$$
\bar{T}\left(x_{1} \gamma_{1}(a)+x_{2} \eta\right)=\gamma_{1}(a) T_{1}\left(x_{1}, x_{2}\right)+\eta T_{2}\left(x_{1}, x_{2}\right),
$$


$T_{1}, T_{2}$ are defined by (4.6). It is clear to see that $r(\bar{T})=r(T)$ holds. From (5.10), if $P\left(i^{*}\right)(a)=$ $0, v^{*}=0, \lambda=0$, we have $r\left(T_{0}\right)=r(\bar{T})=r(T)$.

So, we obtain the following results.

If $r(T)<1$, then $\sup \left\{\operatorname{Re} \lambda, r\left(T_{\lambda}\right)=1\right\}=\lambda_{0}<0$. If $r(T)>1$, then exists $\lambda$ such that $r\left(T_{\lambda}\right)=$ 1 and $\operatorname{Re} \lambda>0$.

From (4.5), for $\left(x_{1}, x_{2}\right)^{T} \in R_{+}^{2}$, we define

$$
\begin{aligned}
\bar{F}\left(x_{1} \gamma_{1}(a)+\eta x_{2}\right)= & \mu^{*} N \gamma_{1}(a) \int_{0}^{+\infty}\left(x_{1} \gamma_{1}(\tau)+\eta x_{2}\right) e^{-\int_{0}^{\tau}\left(x^{*} \gamma_{1}(s)+\eta y^{*}\right) d s} \phi_{1}(\tau) d \tau \\
& +\frac{\eta \delta \mu^{*} \int_{0}^{+\infty}\left(x_{1} \gamma_{1}(\tau)+\eta x_{2}\right) e^{-\int_{0}^{\tau}\left(x^{*} \gamma_{1}(s)+\eta y^{*}\right) d s} \phi_{2}(\tau) d \tau}{\delta \mu^{*} \int_{0}^{+\infty}\left(x^{*} \gamma_{1}(\tau)+\eta y^{*}\right) e^{-\int_{0}^{\tau}\left(x^{*} \gamma_{1}(s)+\eta y^{*}\right) d s} \phi_{2}(\tau) d \tau+\bar{\mu}}
\end{aligned}
$$

where $\left(x^{*}, y^{*}\right)^{T}$ is a nontrivial positive solution of $(x, y)^{T}=F(x, y)$. We easily get that $\bar{F}$ is a nonsupporting operator, and that $r\left(T_{0}\right)<r(\bar{F})$ holds. On the other hand, since $\left(x^{*}, y^{*}\right)^{T}$ is a nontrivial positive solution of $(x, y)^{T}=F(x, y)$, it implies that $\bar{F}$ has a positive eigenfunction $x^{*} \gamma_{1}(a)+\eta y^{*}$ corresponding to eigenvalue 1. Since a nonsupporting operator has only one positive eigenfunction corresponding to its spectral radius, we get that $r(\bar{F})=1$ and $r\left(T_{0}\right)<1$.

From above arguments, we conclude that.

LEMmA 5.5. If the inequality (4.17) holds, under Assumption 5.1, the following results hold:

(1) Suppose that $\left(x^{*}, y^{*}\right)^{T}$ is a trivial solution of $(x, y)^{T}=F(x, y)$.

If $r(T)<1$, then $\sup \left\{\operatorname{Re} \lambda, r\left(T_{\lambda}\right)=1\right\}<0$.

If $r(T)>1$, there exists $\lambda, r\left(T_{\lambda}\right)=1$, Re $\lambda>0$.

(2) Suppose $\left(x^{*}, y^{*}\right)^{T}$ is a nontrivial positive solution of $(x, y)^{T}=F(x, y)$, if $r(T)>1$, then $\sup \left\{\operatorname{Re} \lambda, r\left(T_{\lambda}\right)=1\right\}<0$.

Next, we prove that the operator $B+C$ generates a quasi-compact $C_{0}$-semigroup. We first make the following assumption.

Assumption 5.6. Suppose $\gamma_{1}(a), i^{*}(a)$, p satisfy the condition

$$
\sup _{a \in[0,+\infty)} \gamma_{1}(a) \bar{P}\left(i^{*}\right)<\mu_{0}+p
$$

where $\mu_{0}=\inf _{a \in[0,+\infty)} \mu(a)$.

There exist $\gamma_{1}(\theta)$, p such that Assumption 5.6 holds. In fact, from (4.4) we obtain that $x=\bar{P}\left(i^{*}\right)$ satisfies

$$
\begin{gathered}
x=\mu^{*} N \int_{0}^{+\infty}\left(x \gamma_{1}(\tau)+\eta y\right) e^{-\int_{0}^{\tau}\left(x \gamma_{1}(s)+\eta y\right) d s} \phi_{1}(\tau) d \tau, \\
\phi_{1}(\tau)=e^{(\alpha-p) \tau} \int_{\tau}^{+\infty} \gamma_{2}(a) e^{-\int_{0}^{a}(\mu(s)+\alpha) d s} d a .
\end{gathered}
$$

So we get

$$
z=\gamma_{1}(a) \mu^{*} N \int_{0}^{+\infty}(z(\tau)+\eta y) e^{-\int_{0}^{\tau}(z(s)+\eta y) d s} \phi_{1}(\tau) d \tau
$$


where $z=\gamma_{1}(a) \bar{P}\left(i^{*}\right)$. From above equation, if $\gamma_{1}(a)$ is small enough, we easily obtain that $\gamma_{1}(a) \bar{P}\left(i^{*}\right)$ may be small enough such that Assumption 5.6 holds.

In order to investigate the stability of equilibria we prove the following lemma.

Lemma 5.7. The operator $B$ is a closed linear operator and exists $\varepsilon>0$ such that $\lambda-B$ has bounded inverse for $\lambda>-\varepsilon$ and

$$
\left\|(\lambda-B)^{-n}\right\| \leq \frac{1}{(\lambda+\varepsilon)^{-n}} .
$$

Proof. For $f=\left(f_{1}, f_{2}, f_{3}\right)^{T} \in X_{+}$, consider the equation $(\lambda-B) u=f$ with $\lambda>-\varepsilon$, we have

$$
\begin{gathered}
\frac{d u_{1}(a)}{d a}=-\left(\lambda+P\left(i^{*}\right)(a)+\eta v^{*}+p+\mu(a)\right) u_{1}+f_{1}, \\
\frac{d u_{2}(a)}{d a}=\left(P\left(i^{*}\right)(a)+\eta v^{*}\right) u_{1}-(\lambda+\alpha+\mu(a)) u_{2}+f_{2}, \\
0=-\left(\lambda+\delta H\left(i^{*}\right)+\bar{\mu}\right) u_{3}+f_{3}, \\
u_{1}(0)=0, \quad u_{2}(0)=0 .
\end{gathered}
$$

From (5.29), we obtain

$$
\begin{aligned}
& u_{1}(a)=e^{-\int_{0}^{a}\left(\lambda+P\left(i^{*}\right)(s)+\eta v^{*}+p+\mu(s)\right) d s} \int_{0}^{a} f_{1}(\tau) e^{\int_{0}^{\tau}\left(\lambda+P\left(i^{*}\right)(\tau)+\eta v^{*}+p+\mu(\tau)\right) d \tau} d \tau, \\
& u_{2}(a)=e^{-\int_{0}^{a}(\lambda+\alpha+\mu(s)) d s} \int_{0}^{a}\left[\left(P\left(i^{*}\right)(\tau)+\eta v^{*}\right) u_{1}(\tau)+f_{2}(\tau)\right] e^{\int_{0}^{\tau}(\lambda+\alpha+\mu(s)) d s} d \tau, \\
& u_{3}=\frac{f_{3}}{\lambda+\delta^{*}} \\
& \|u\| \leq \frac{1}{\left(\lambda+\lambda^{*}+\eta v^{*}+p+\mu_{0}\right)}\left\|f_{1}\right\| \\
& +\frac{P^{*}+\eta v^{*}}{\left(\lambda+\alpha+\mu_{0}\right)\left(\lambda+\lambda^{*}+\eta v^{*}+p+\mu_{0}\right)}\left\|f_{1}\right\|+\frac{1}{\lambda+\alpha}\left\|f_{2}\right\| \\
& +\frac{1}{\lambda+\delta^{*}}\left\|f_{3}\right\|
\end{aligned}
$$

where $\lambda^{*}=\inf _{0 \leq a<\infty} P\left(i^{*}\right)(a), P^{*}=\sup _{0 \leq a<\infty} P\left(i^{*}\right)(a)$.

From Assumption 5.6, as $\varepsilon$ (which depends on $\eta, \alpha, \delta, \mu_{0}, \bar{\mu}, i^{*}$ ) is sufficiently small we can obtain

$$
\|u\| \leq \frac{1}{\lambda+\varepsilon}\left(\left\|f_{1}\right\|+\left\|f_{2}\right\|+\left\|f_{3}\right\|\right) .
$$

If $f \in X$, then $\left\|(\lambda-B)^{-1} f\right\| \leq\left\|(\lambda-B)^{-1}|f|\right\|$. Consequently, the claim follows.

It is clear that $B$ in $X$ is a densely defined operator whose resolvent satisfies the HilleYosida estimate, and is the generator of a $C_{0}$-semigroup on $X, S(t)$ (see [12]). The HilleYosida estimate in addition implies that

$$
\|S(t)\| \leq e^{-\varepsilon t} .
$$


On the other hand, $C$ is a bounded perturbation and $B+C$ also generates a $C_{0}$-semigroup on $X, J(t)$. Furthermore, since $C$ is a compact operator $C S(t): X \rightarrow X$ is also compact for every $t>0$. So, all conditions of part (b), [14, Theorem 3] are satisfied and $J(t)$ is quasicompact.

Quasi-compact is defined as following.

Definition 5.8. $J(t)$ is called quasi-compact if $J(t)=J_{1}(t)+J_{2}(t)$ with operator families $J_{1}(t), J_{2}(t)$, where $\left\|J_{1}(t)\right\| \rightarrow 0$, as $t \rightarrow 0, J_{2}(t)$ is eventually compact, that is, there exists $t_{0}>0$ such that $J_{2}(t)$ is a compact operator for all $t>t_{0}$.

To establish the local stability we use the following lemma which is a special case of [1, Theorem 2.10, Chapter B-IV].

Lemma 5.9. Let $J(t)$ be a quasi-compact $C_{0}$-semigroup and $\bar{B}$ its infinitesimal generator. Then $e^{\omega t}\|J(t)\| \rightarrow 0$ as $t \rightarrow+\infty$ for $\omega>0$ if and only if all eigenvalues of $\bar{B}$ have strictly negative real part.

From Lemmas 5.5 and 5.9, we have the following theorem.

Theorem 5.10. If the inequality (4.17) holds, under Assumptions 5.1 and 5.6, the following results hold:

(1) If $r(T)<1$, then the trivial equilibrium point of the system (2.6)-(2.8) is locally asymptotically stable.

(2) If $r(T)>1$, then the trivial equilibrium point of the system (2.6)-(2.8) is unstable.

(3) If $r(T)>1$, then the endemic equilibrium point of the system (2.6)-(2.8) is locally asymptotically stable.

\section{Acknowledgments}

The authors thank Professor H. Nijmeijer and two reviewers for their kind suggestions and careful corrections to our previous manuscript which were most helpful in improving it.

\section{References}

[1] W. Arendt, A. Grabosch, G. Greiner, U. Groh, H. P. Lotz, U. Moustakas, R. Nagel, F. Neubrander, and U. Schlotterbeck, One-Parameter Semigroups of Positive Operators, Lecture Notes in Mathematics, vol. 1184, Springer, Berlin, 1986.

[2] Z. Feng, D. L. Smith, F. E. McKenzie, and S. A. Levin, Coupling ecology and evolution: Malaria and the S-gene across time scales, Math. Biosci. 189 (2004), no. 1, 1-19.

[3] S. Gupta and A. V. Hill, Dynamic interactions in malaria: Host heterogeneity meets parasite polymorphism, Proc. R. Soc. Lond. B Biol. Sci. 261 (1995), no. 1362, 271-277.

[4] H. J. A. M. Heijmans, The dynamical behaviour of the age-size-distribution of a cell population, The Dynamics of Physiologically Structured Populations (Amsterdam, 1983) (J. A. J. Metz and O. Diekmann, eds.), Lecture Notes in Biomath., vol. 68, Springer, Berlin, 1986, pp. 185-202.

[5] H. Inaba, Threshold and stability results for an age-structured epidemic model, J. Math. Biol. 28 (1990), no. 4, 411-434. 
[6] Kermack and McKendrick revisited: The variable susceptibility model for infectious diseases. Recent topics in mathematics moving toward science and engineering, Japan J. Indust. Appl. Math. 18 (2001), no. 2, 273-292.

[7] H. Inaba and H. Sekine, A mathematical model for Chagas disease with infection-age-dependent infectivity, Math. Biosci. 190 (2004), no. 1, 39-69.

[8] M. A. Krasnosel'skiĭ, Positive Solutions of Operator Equations, Noordhoff, Groningen, 1964.

[9] X.-Z. Li, G. Gupur, and G.-T. Zhu, Threshold and stability results for an age-structured SEIR epidemic model, Comput. Math. Appl. 42 (2001), no. 6-7, 883-907.

[10] I. Marek, Frobenius theory of positive operators: Comparison theorems and applications, SIAM J. Appl. Math. 19 (1970), no. 3, 607-628.

[11] J. Müller, Optimal vaccination patterns in age-structured populations, SIAM J. Appl. Math. 59 (1999), no. 1, 222-241.

[12] A. Pazy, Semigroups of Linear Operators and Applications to Partial Differential Equations, Applied Mathematical Sciences, vol. 44, Springer, New York, 1983.

[13] I. Sawashima, On spectral properties of some positive operators, Natur. Sci. Rep. Ochanomizu Univ. 15 (1964), 53-64.

[14] H. R. Thieme, Quasi-compact semigroups via bounded perturbation, Advances in Mathematical Population Dynamics-Molecules, Cells and Man (Houston, Tex, 1995) (O. Arino, D. Axelrod, and M. Kimmel, eds.), Ser. Math. Biol. Med., vol. 6, World Scientific, New Jersey, 1997, pp. 691-711.

[15] H. R. Thieme and C. Castillo-Chavez, How may infection-age-dependent infectivity affect the dynamics of HIV/AIDS? SIAM J. Appl. Math. 53 (1993), no. 5, 1447-1479.

[16] G. F. Webb, Theory of Nonlinear Age-Dependent Population Dynamics, Monographs and Textbooks in Pure and Applied Mathematics, vol. 89, Marcel Dekker, New York, 1985.

Helong Liu: Department of Mathematics, Xinyang Normal University, Xinyang, Henan 464000, China

Current address: Beijing Institute of Information and Control, Beijing 100037, China

E-mail address: liuhelong2004@yahoo.com.cn

Houbao Xu: Department of Mathematics, Beijing Institute of Technology, Beijing 100081, China

E-mail address: xuhoubao@yahoo.com.cn

Jingyuan Yu: Beijing Institute of Information and Control, Beijing 100037, China

E-mail address: yujingyuan@biic.net

Guangtian Zhu: Academy of Mathematics and System Science, Chinese Academy of Sciences, Beijing 100080, China

E-mail address: gtzhu@amss.ac.cn 


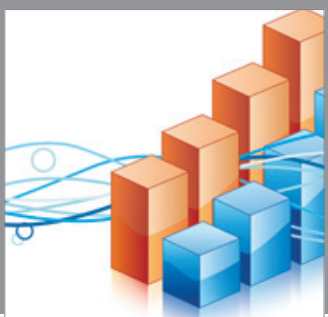

Advances in

Operations Research

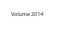

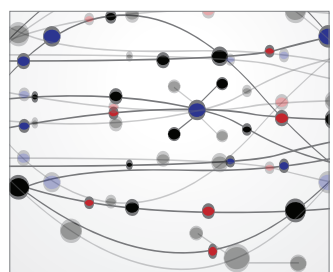

\section{The Scientific} World Journal
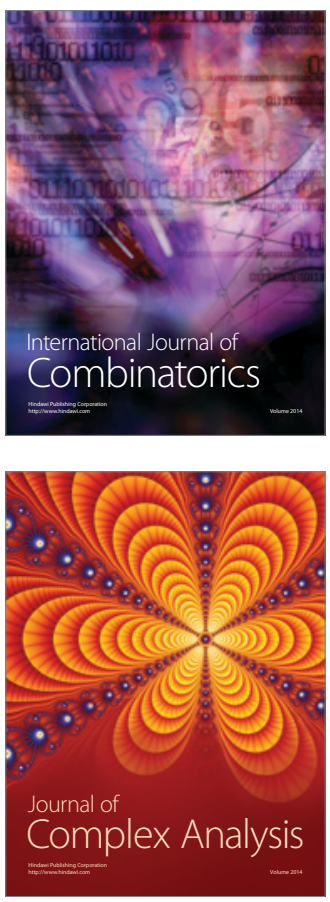

International Journal of

Mathematics and

Mathematical

Sciences
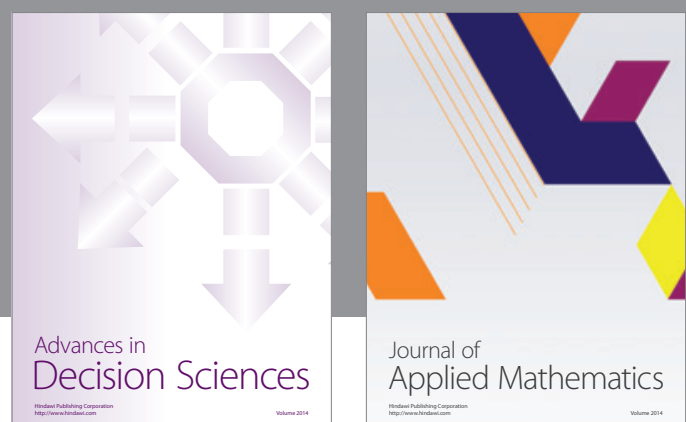

Journal of

Applied Mathematics
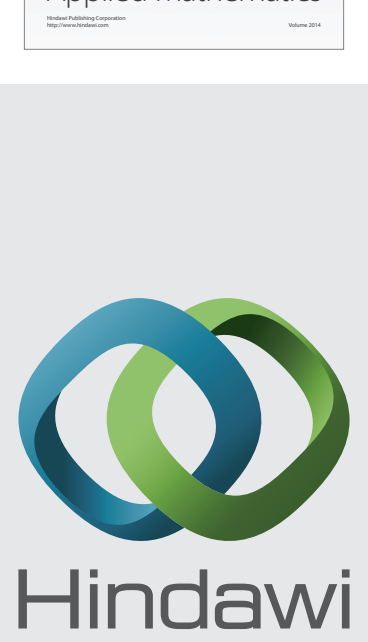

Submit your manuscripts at http://www.hindawi.com
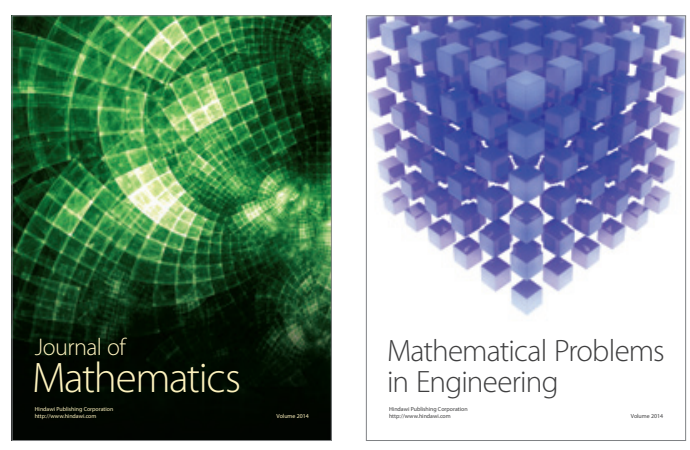

Mathematical Problems in Engineering
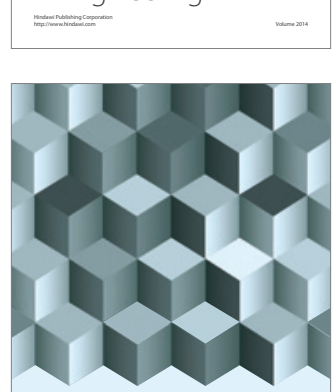

Journal of

Function Spaces
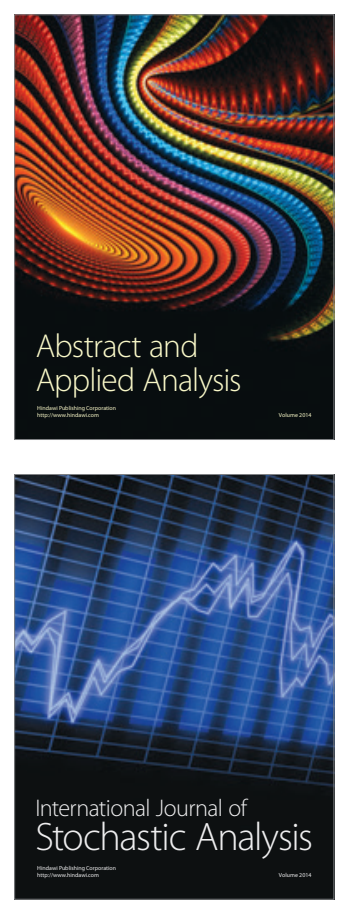

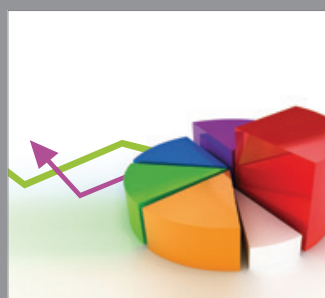

ournal of

Probability and Statistics

Promensencen
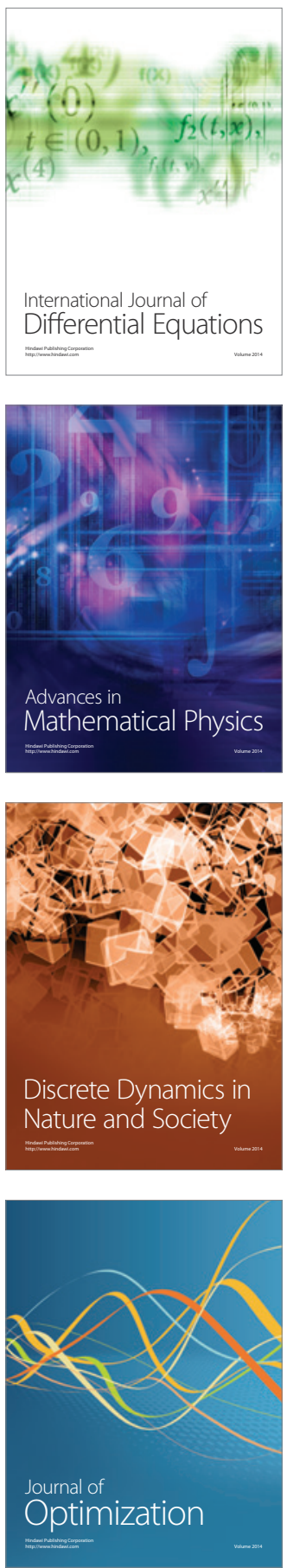